\title{
Le contrôle antidopage des animaux, nouvelles perspectives, nouvelles méthodes
}

\author{
Animal doping control, new aspects, \\ new methods
}

Yves BONNAIRE, Marie-Agnès POPOT, Louis DEHENNIN

L.A.B.-F.N.C.F., 169, avenue de la division Leclerc - 92290 CHATENAY MALABRY

(Reçu le 2 décembre 1999 ; accepté le 13 janvier 2000)

\section{RÉSUMÉ}

Les nouvelles méthodes de détection pouvant être mises en place dans les laboratoires sont principalement axées autour des spectromètres de masse de haute résolution, notamment les appareils de temps de vol (TOF) dont la technologie s'est considérablement améliorée ces dernières années. Ces techniques sont susceptibles d'apporter à la fois une meilleure sensibilité et spécificité. En effet l'accroissement de résolution. permet d'augmenter significativement le rapport signal/bruit et dans la mesure où la sensibilité de l'appareil n'est pas altérée en conséquence, le gain en terme de capacité de détection est donc important. Dans le même esprit, de nouveaux couplages de chromatographie liquide capillaire/MS/MS, alliant à la fois la résolution chromatographique des colonnes "micro ou nanobore" aux performances des spectromètres en tandem (MS/MS) récents ou des trappes d'ions permettent d'améliorer significativement les limites de détection des xénobiotiques dopants.

Des exemples de détection de stérö̈des anabolisants dans les milieux biologiques équins illustrent ces nouvelles perspectives analytiques.

Le second axe de développement s'articule autour de la prise en compte de marqueurs biologiques qui peuvent être altérés par l'administration de biomolécules telles que les hormones de croissance par exemple. Le problème principal posé par l'administration de ces peptides réside dans le fait que ces molécules elles-mêrme sont difficiles à détecter du fait de leur poids moléculaire élevé et surtout par le fait que leur demi-vie est très courte. Ces deux phénomènes ont pour conséquence de rendre aléatoire la mise en évidence de la molécule elle-même, une solution possible à cela consiste à prendre en compte les marqueurs biologiques altérés par l'administration de l'hormone protéique. Ainsi un exemple basé sur le suivi de ces marqueurs et en particulier de l'IGFl, après administration d'hormone de croissance recombinante au cheval est exposé.

\section{MOTS-CLES}

Dopage, Chromatographie Gazeuse Spectrométrie de Masse, Anabolisant, Hormone de Croissance.

\section{SUMMARY}

Dope testing laboratories need improved sensitivity for some specific drugs such as anabolic steroids. New generation high resolution mass spectrometers, such as Time Of Flight (TOF) or even magnetic sectors systems, can improve dramatically the sensitivity of detection by improving the signal to noise ratio through increase of resolution without decrease of sensitivity.

In the same spirit micro or nanobore capillary liquid chromatography coupled with MS/MS (quadrupoles or ion trap) can lower significantly the limit of detection of some drugs. Examples involving anabolic steroid detection in horse urine are shown.

Another extension of the dope testing concept needs to be taken into account. Biological macromolecules such as erythropoietin (EPO) or growth hormone (GH) can be monitored by their endogenous markers. The half-life of such peptides is so short that their detection in biological fluids remains uncertain. Therefore, the monitoring of such molecules is far more easier by analysing the effect on the biomarkers, significantly altered by the drug. An example of the variation of plasmatic IGFl by administration of recombinant $G H$ in the horse is given.

\section{KEY-WORDS}

Dope testing, Gas Chromatography, Mass Spectrometry, Anabolic Steroid, Growth Hormone. 


\section{Introduction}

Les animaux de sport ou de course sont soumis, comme tous les athlètes à des thérapies variées visant soit à restaurer leurs capacités propres, soit à les améliorer artificiellement. Depuis de très nombreuses années, le contrôle antidopage est mis en place, en France comme dans de le monde entier. Ce contrôle a montré clairement son efficacité mais aussi ses limites, il convient donc de l'adapter à la fois aux nouvelles molécules, mais aussi aux nouvelles pratiques dopantes. Ces évolutions impliquent à la fois une amélioration des processus de détection employés dans les laboratoires de contrôle antidopage, mais aussi une extension du concept du contrôle antidopage lui même.

\section{Évolution des méthodes et des appareillages de contrôle.}

Ces dernières décennies ont connu l'évolution, voire la révolution dans le choix des méthodes analytiques utilisées pour le contrôle antidopage. La disparition des tests à base de chromatographie sur couche mince au profit des chromatographies en phase gazeuse, puis liquide de haute performance et l'apparition de la spectrométrie de masse ont fait considérablement progresser les performances des tests de contrôle antidopage. Néanmoins, les nouvelles molécules et surtout les nouvelles formes d'administration des agents dopants, comme les cocktails ou l'administration de certaines molécules par voie pulmonaire, impose une sensibilité accrue des tests de dépistage.

Parmi les molécules visées par cet abaissement nécessaire du niveau de détection, les stéroïdes anabolisants figurent en tête de liste. Dans cette classe pharmacologique figure une molécule plus particulièrement délicate à détecter : il s'agit du stanozolol, qui certes n'est pas nouveau, mais sa détection dans les milieux biologiques (1) est difficile pour deux raisons principales. La première est liée à un métabolisme complexe, aboutissant à plusieurs métabolites hydroxylés présents à faible ccncentration ; le second obstacle rencontré est lié aux difficultés chromatographiques associées à la séparation des dérivés silylés du stanozolol et de ses métabolites.

Nous avons ainsi voulu évaluer, à partir d'échantillons biologiques réels obtenus par administration de cette substance au cheval, les améliorations apportées par l'usage de spectromètres de masse haute résolution (2) ou de couplages faisant appel à la chromatographie liquide de haute performance (3), couplée à la spectrométrie de masse de haute résolution TOF, ou même hybride (Q-TOF), comparativement aux instruments de basse résolution employés quotidiennement dans les laboratoires de contrôle.

\section{Matériel et méthodes. Instruments :}

Les instruments employés lors de cette étude sont les suivants :

1. GC-MS, appareil de basse résolution quadripolaire (Hewlett Packard 5973) ; colonne capillaire HP-5 $(25 \mathrm{~m} / 0.25 \mathrm{~mm} / 0.2 \mu \mathrm{m})$, splitless, $2 \mu \mathrm{L}$ injectés sur $30 \mu \mathrm{l}$ de réactif .

2. GC-HRMS, appareil magnétique, résolution 10000 (MAT 95, Finnigan THERMOQUEST) ; colonne UItra1 $(17 \mathrm{~m} / 0.20 \mathrm{~mm} / 0.11 \mu \mathrm{m})$, split $1 / 20,2 \mu \mathrm{l}$ sur $30 \mu \mathrm{L}$ de réactif injectés.

3. LC-Q-TOF, (quadripole + temps de vol), résolution 5500 , source electrospray (Micromass) ; colonne 150 x $2 \mathrm{~mm}, 5 \mu \mathrm{m}, \mathrm{C} 18$, methanol-eau, $150 \mu \mathrm{L} / \mathrm{mn}, 20 \mu \mathrm{l}$ sur $400 \mu \mathrm{L}$ de solvant de reprise injectés.

4. $\mu$ HPLC-MS, triple quadripole, source electrospray (Perkin Elmer Sciex 365) ; colonne $50 \times 0.3 \mathrm{~mm}, 3 \mu \mathrm{m}$, C18, acétonitrile-eau- $0.1 \%$ acide formique, $4 \mu \mathrm{L} / \mathrm{mn}$, $5 \mu l$ sur $400 \mu \mathrm{L}$ de reprise injectés.

5. LC-TOF, temps de vol, résolution 5000, source electrospray (Mariner API-TOF, PE Biosystems) ; colonne $150 \times 1 \mathrm{~mm}, \mathrm{C} 18$, methanol-eau- $0.2 \%$ acide formique$4 \mathrm{mM}$ acétate d'ammonium, $50 \mu \mathrm{L} / \mathrm{mn}, 5 \mu \mathrm{l}$ sur $200 \mu \mathrm{L}$ de solvant injectés.

\section{Protocole expérimental}

Une dose de stanozolol (Sigma ref S7132) à raison de $0.8 \mathrm{mg} / \mathrm{kg}$ a été administrée par voie orale à un cheval pur sang mâle, les urines ont été collectées pendant $72 \mathrm{~h}$. Deux mélanges (pool) urinaires de $150 \mathrm{~mL}$ ont été constitués :

- taux moyen (environ $3 \mathrm{ng} / \mathrm{mL}$, urine de $24 \mathrm{~h}$ après administration) ;

- taux faible (environ $1 \mathrm{ng} / \mathrm{mL}$, urines de $36 \mathrm{~h}$ et $48 \mathrm{~h}$ après administration).

\section{Préparation de l'échantillon :}

Cinq $\mathrm{mL}$ d'urine ont été extraits en phase solide (cartouche $\mathrm{C} 18$ ) avec élution par le méthanol. Les métabolites glucuro- et les sulfoconjugués ont été séparés par chromatographie d'échange d'ions (DEAE-Sephadex). Les glucuronides ont été hydrolysés par la $\beta$-glucuronidase et les sulfates ont été déconjugués par méthanolyse acide. Les métabolites libres ont été extraits à $\mathrm{pH} 9$ avec de l'éther éthylique et les deux fractions ont ensuite été mélangés. Pour l'analyse $\mathrm{GC}$ le dérivé bis(triméthylsilyle) a été formé avec $30 \mu \mathrm{L}$ de réactif silylant.

Un témoin contenant $200 \mathrm{ng}$ de stanozolol, 3'-hydroxystanozolol et $16 \beta$-hydroxystanozolol a été analysé dans chaque série. 


\section{Résultats Discussion}

Les figures $1 \mathrm{~A}$ (concentration moyenne) et $1 \mathrm{~B}$ (concentration faible) montrent les fragmentogramimes obtenus à basse résolution sur un appareil HP 5973 avec les ions $\mathrm{m} / \mathrm{z} 457$ et 472 caractéristiques du stanozolol, le tracé 1B n'est pas interprétable et est représentatif de la limite de détection qualitative.

Les tracés obtenus sur l'appareil MAT95 sont représentés sur les figures $2 \mathrm{~A}$ et $2 \mathrm{~B}$ où le fragmentogramme $2 \mathrm{~B}$, réalisé sur la faible concentration, rend encore une interprétation possible.

Les analyses réalisées en couplage HPLC/MS semblent prometteuses, les figures $3 \mathrm{~A}$ et $3 \mathrm{~B}$ montrent les résultats obtenus en $\mathrm{LC} / \mathrm{Q} / \mathrm{TOF}$ sur le 16 $\beta$-hydroxystanozolol. Le pic obtenu à partir de la faible concentration est bien individualisé. Il est à remarquer que ces tracés représentent la somme des ions MS/MS obtenus à partir des ions moléculaires en TOF à une résolution de 5500 .

L'analyse par LC/MS/MS sur un triple quadripole représentée sur les figures $4 \mathrm{~A}$ et $4 \mathrm{~B}$ est intéressante car elle fait apparaître le 16 $\beta$-hydroxystanozolol et deux autres dérivés hydroxylés $\mathrm{X}-\mathrm{OH}$ et $\mathrm{Y}-\mathrm{OH}$, dont la

A
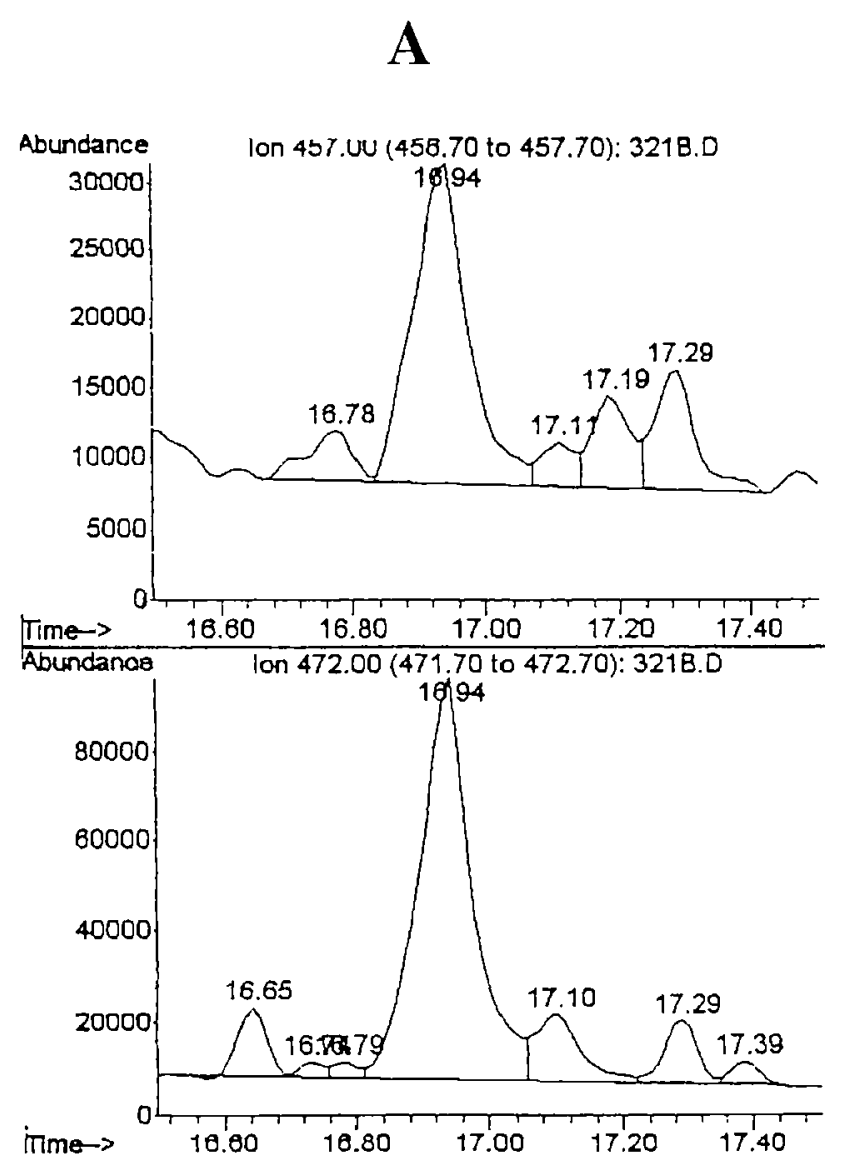

configuration n'est pas entièrement élucidée. La concentration faible montre encore des pics décelables malgré la dilution très importante des extraits chromatographiés.

Enfin les figures $5 \mathrm{~A}$ et $5 \mathrm{~B}$ montrent ces extraits analysés en LC/TOF, seule la concentration moyenne est interprétable.

Les cinq types d'analyse ont permis de détecter le stanozolol et ses métabolites dans le mélange à taux moyen. Dans le mélange à taux faible, la détection n'a été possible que par GC-HRMS magnétique, par couplage HPLC capillaire-triple quadripole et par LC-QTOF (par ordre décroissant de performance).

L'amélioration de la détectabilité du stanozolol dans les extraits urinaires destinés aux analyses de dépistage (screening), avec des instruments plus performants que le système de basse résolution classiquement utilisé en dépistage de routine, reste modeste. Cette performance accrue ne pourra atteindre un seuil réellement significatif qu'avec des extraits ayant subi une étape de purification complémentaire, qui permettront aux systèmes de haute résolution de s'exprimer totalement.

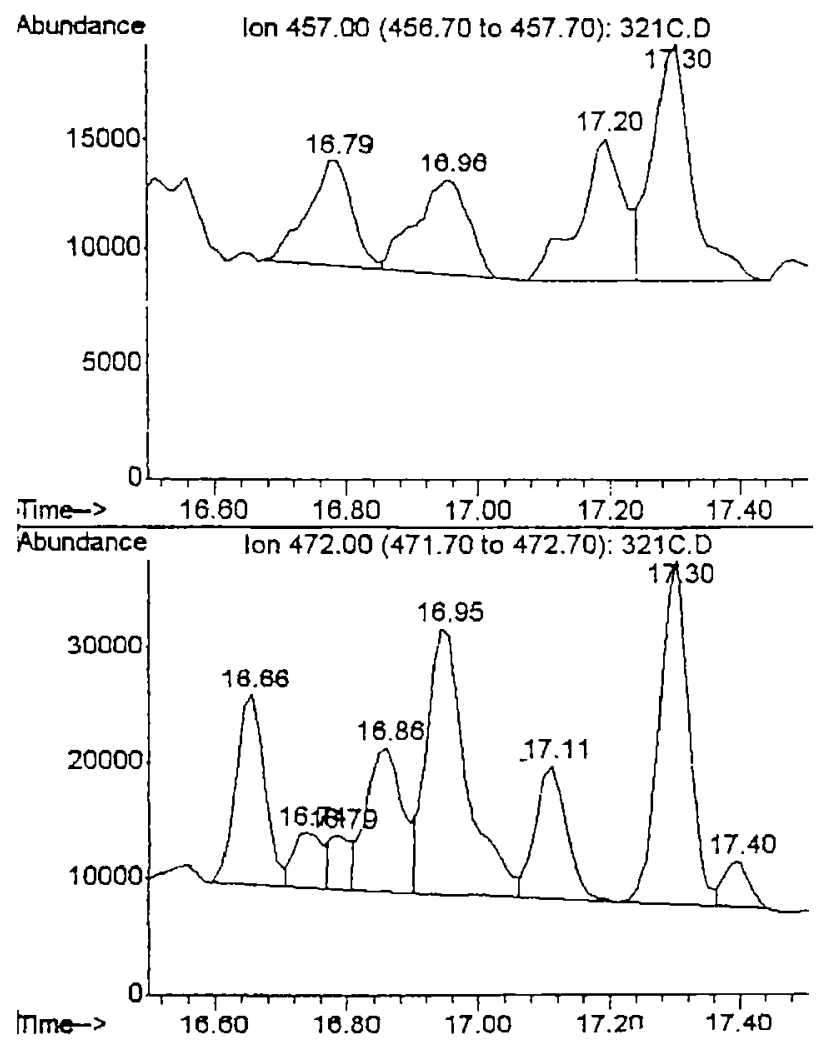

Figures 1A et $1 B$ : GC/MS (HP 5973) 
A

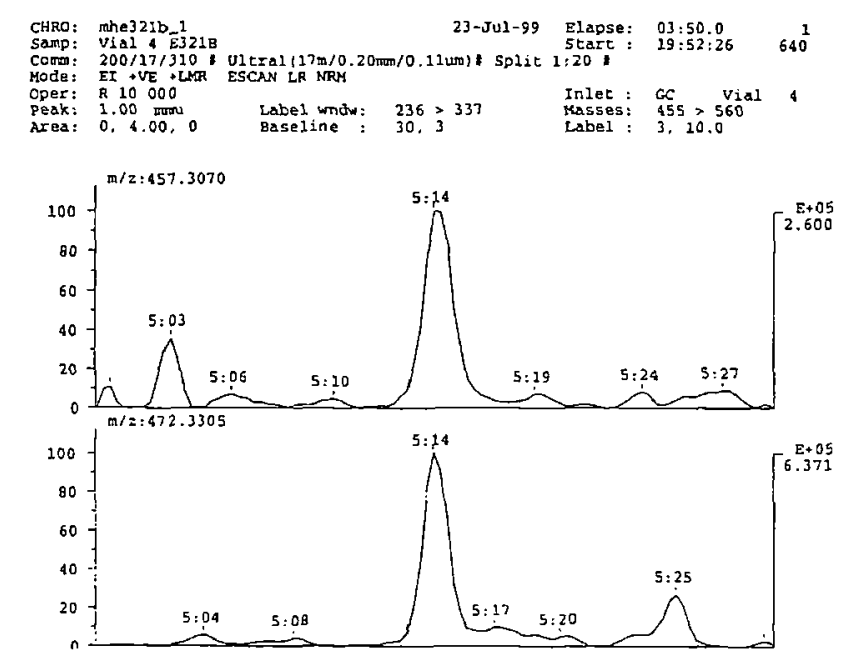

B
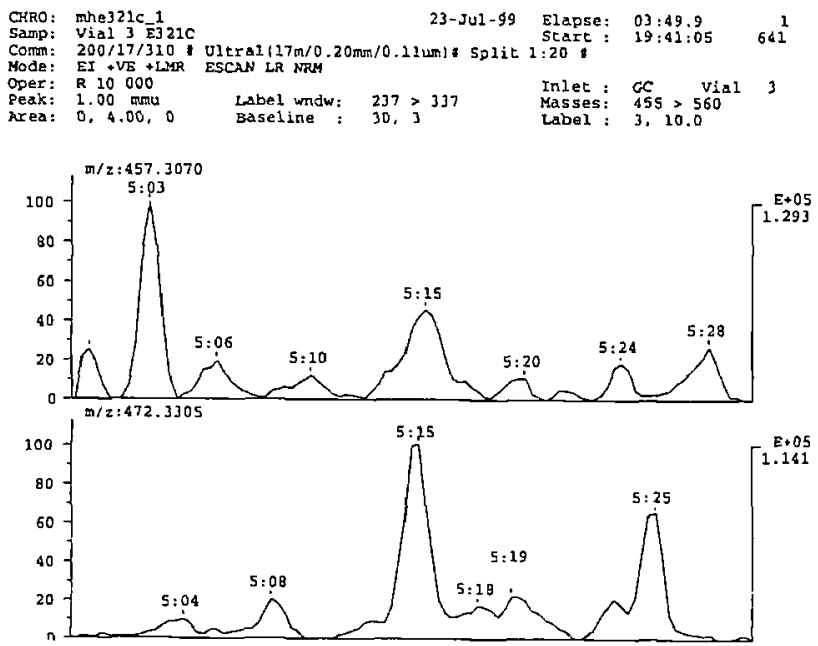

Figures $2 A$ et $2 B: G C / H R M S$ (THERMOQUEST MAT 95)

STANOZOLOL, TR $=5,14 \mathrm{mn}$, ions $\mathrm{m} / \mathrm{z}$ 457,3070 et 472,3305

$A=$ concentration moyenne, $B=$ concentration faible

\section{A}

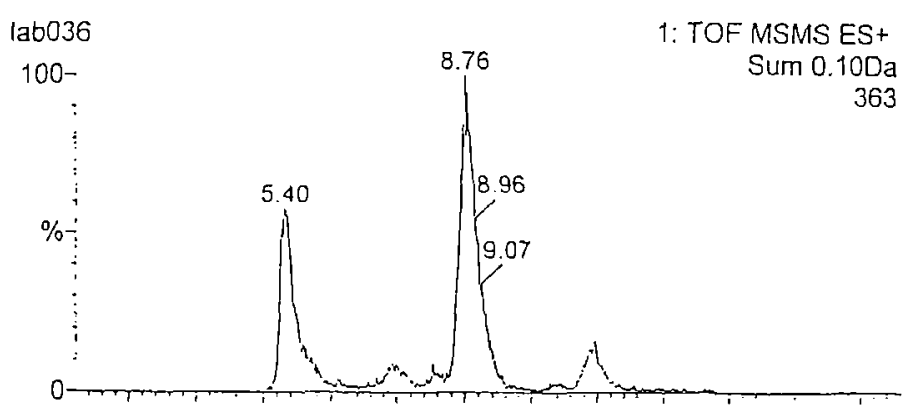

\section{B}

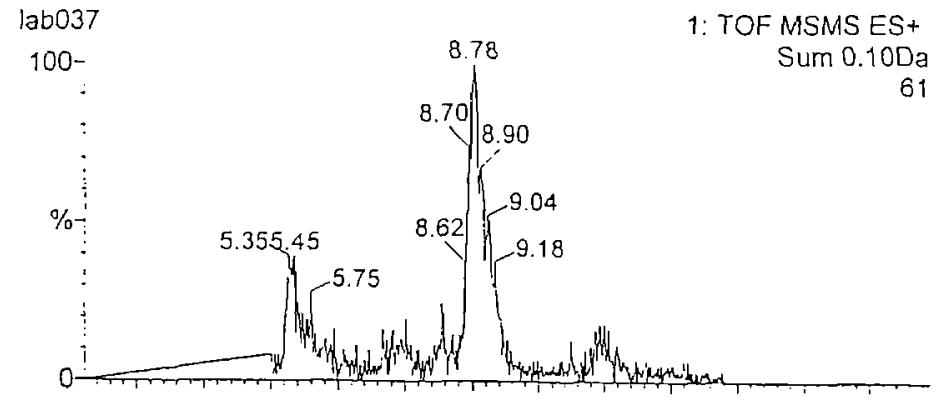

Figures 3A et 3B : LC/Q-TOF (MICROMASS)

$16 \beta$ HYDROXYSTANOZOLOL, TR $=8,76 \mathrm{mn}$ et $8,78 \mathrm{mn}$ ions $\mathrm{m} / \mathrm{z} 345,26 \rightarrow$ somme de $\mathrm{m} / \mathrm{z}(81,058+95,081+121,126)$

$A=$ concentration moyenne, $B=$ concentration faible

\section{A}
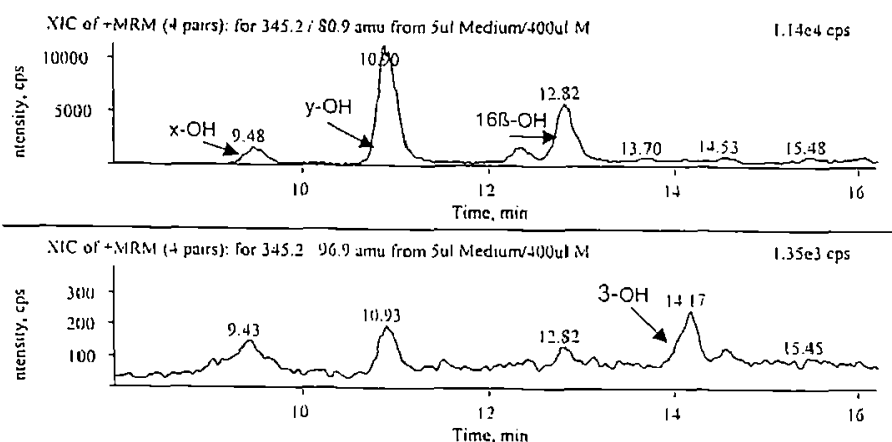

B

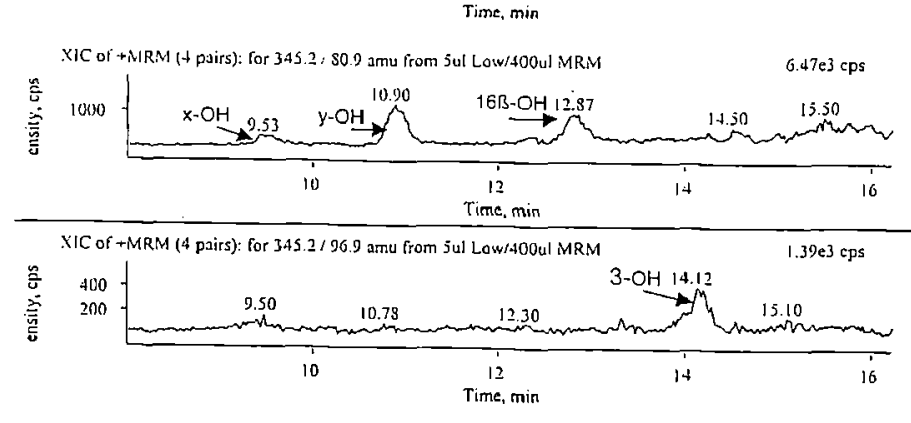

Figures $4 A$ et $4 B$ : COUPLAGE HPLC CAPILLAIRE/MS TRIPLEQUADRIPOLE (PERKIN ELMER SCIEX 365)

HYDROXYSTANOZOLOLS, TR $=9,48 \mathrm{mn}, 10,90 \mathrm{mn}$ et $12,82 \mathrm{mn}$, ions $m / z 345,2 \rightarrow 80,9$ et 96,9

$A=$ concentration moyenne, $B=$ concentration faible 


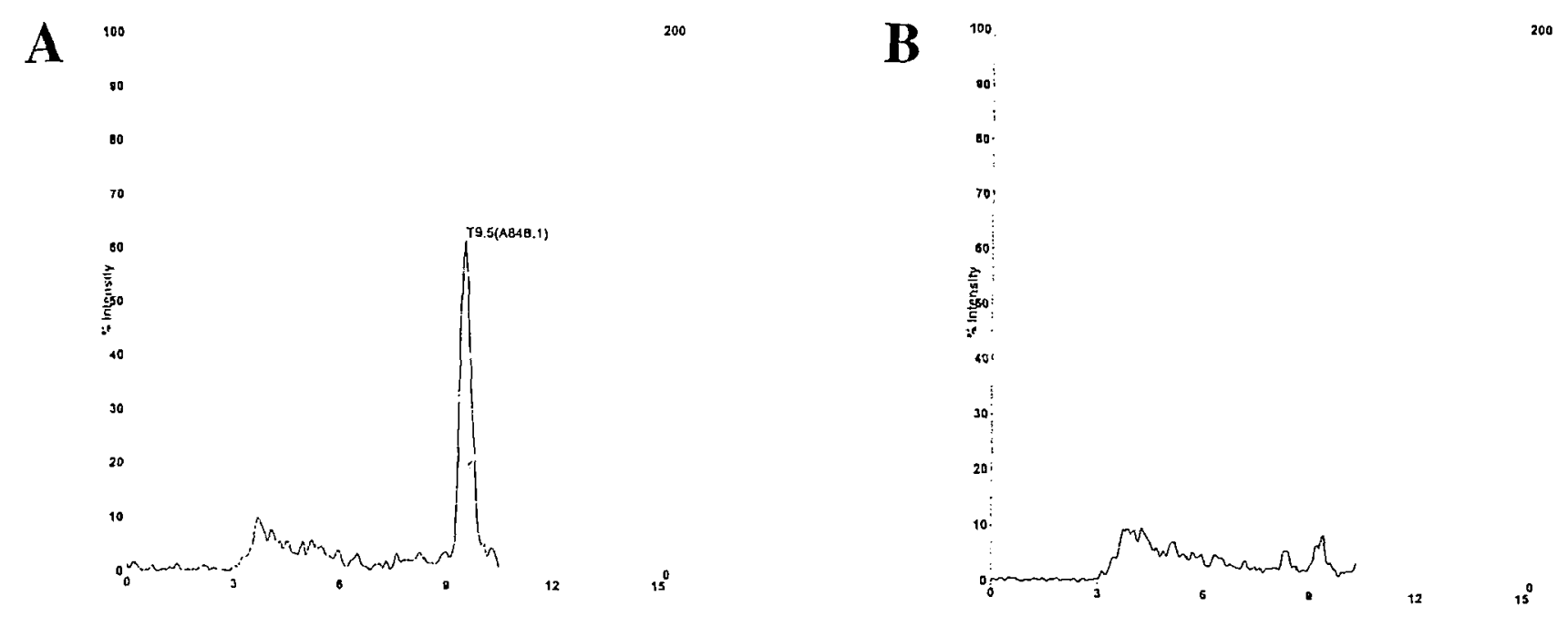

Figures 5A et 5B : (PERKIN ELMER BIOSYSTEM, MARINER)

STANOZOLOL, TR = 9,5 mn, ion $\mathrm{m} / \mathrm{z} 329,2587$

$A=$ concentration moyenne, $B=$ concentration faible

\section{Évolution du concept de substance prohibée.}

Jusqu'à présent seule la mise en évidence de la ou des substances dopantes pouvait être retenue pour matérialiser "un cas positif". Dorénavant l'extension du concept de substance prohibée (article 6 de l'Accord International dur l'élevage et les courses, référence 4) permet de prendre en compte également les "indicateurs scientifiques" prouvant qu'il y a eu administration ou exposition à une substance prohibée. Cette alternative est particulièrement adaptée au cas des hormones peptidiques, telles que l'EPO ou l'hormone de croissance.

Du fait de son action trophique, l'utilisation de l'hormone de croissance est hautement soupçonnée chez les athlètes humains (5). En effet, elle est souvent considérée comme plus active que les stéroïdes anabolisants. De plus, certains athlètes et culturistes l'utiliseraient en association avec les anabolisants pour augmenter la masse musculaire et la force physique.

La détection de son administration doit aussi être une préoccupation des analystes des courses de chevaux. En raison de la demi-vie courte et de la sécrétion pulsatile et accrue après effort physique de l'hormone endogène (6), l'analyse de l'hormone de croissance recombinante équine native ne peut pas résoudre le problème de la détection de son administration. Par contre la quantification de marqueurs endogènes de son administration peuvent constituer la solution. Parmi ces marqueurs, il faut citer l'IGF1, les protéines de transport de 1'IGF1 et certains marqueurs osseux. En effet au niveau périphérique, les effets physiologiques de l'hormone de croissance s'exercent par l'intermédiaire du foie qui produit l'IGF1.

L' IGF1 qui est l'exemple que nous développerons ici, a été étudiée précédemment chez l'homme par Lee et al. (7) et Kicman et al. (5). Lee et al. (7) ont montré l'élévation de l'IGF1 chez des malades traités au long cours par l'hormone de croissance recombinante humaine.

Dans l'étude de Kicman et al. (5), des volontaires sains ont reçu pendant trois jours de l'hormone de croissance recombinante humain. Une augmentation significative de l'IGF1 a été enregistrée. Ils ont ainsi suggéré de prendre en compte ce marqueur pour développer un test de détection de l'administration de l'hormone de croissance chez les sportifs. Chez le cheval, les valeurs basales de ce paramètre ont fait l'objet de quelques déterminations. Malinowski et al. (8) ont étudié les effets de l'âge et de la race chez des juments de 1 à 18 ans. La concentration en IGF1 plasmatique varie avec l'âge, augmentant du jour de la naissance jusqu'au $9^{\text {eme }}$ mois pour diminuer ensuite, ces observations étant en accord avec ce qui a été observé chez l'homme et les bovins. Thomas et al. (9) ont déterminé les concentrations plasmatiques d'hormone de croissance et d'IGF1 chez des jeunes chevaux "quater horse" et des poneys et ils n'ont pas trouvé de différences entre les deux races.

Dans la présente étude, on se propose d'étudier les concentrations plasmatiques d'IGF1 dans une population de chevaux surveillée et les évolutions de sa concentration plasmatique après administration d'hormone de croissance chez deux chevaux. En ce qui concerne les valeurs basales nous avons étudié les variations de la concentration plasmatique chez trois chevaux prélevés régulièrement. 


\section{Matériel et méthode}

\section{Variation nycthémérale de la concentration.}

Trois chevaux (un mâle, un hongre et une femelle) ont été sélectionnés pour cette étude. Des échantillons ont été obtenus toutes les heures de $5 \mathrm{~h}$ à $21 \mathrm{~h}$.

\section{Étude de population.}

Des chevaux (170) de l'Institut du Cheval à Pompadour, âgés de 1 à 26 ans, ont été sélectionnés pour cette étude. L'âge et le sexe étaient connus pour tous, la race était connue pour bon nombre d'entre eux.

\section{Administration d'hormone recombinante équine à deux chevaux.}

L'hormone "Bresagen" recombinante équine lyophilisée a été utilisée pour cette expérimentation et reconstituée dans de l'eau distillée en une solution à $2,5 \mathrm{mg} / \mathrm{ml}$.

Deux chevaux de 10 et 11 ans ont reçu pendant 14 jours des injections journalières de cette solution à la dose de $18 \mu \mathrm{g} / \mathrm{kg}$ la première semaine et de $25 \mu \mathrm{g} / \mathrm{kg}$ la deuxième semaine. Des prélèvements sont faits un, deux et trois jours avant administration, ensuite tous les jours pendant 35 jours, et enfin 45 , 55, et 60 jours après administration.

\section{Type de prélèvements :}

Les prélèvements sanguins ont été faits dans des tubes contenant de l'héparinate de lithium. Les prélèvements ont ensuite été centrifugés à 2000 tours/mn pendant 20 minutes à température ambiante. Les plasmas sont prélevés immédiatement et congelés jusqu'au moment de l'analyse.

\section{Méthode analytique}

L'IGF1 totale a été dosé par dosage radioimmunométrique (IRMA) à l'aide d'une trousse du Laboratoire Biocode (Liége, Belgique).

Cent $\mu \mathrm{l}$ d'échantillon sont préalablement extraits à l'éthanol acide, neutralisé par un tampon TRIS et sont soumis au dosage. Une aliquote de $25 \mu \mathrm{L}$ de la solution d'extraction sont utilisés pour le dosage. Chaque échantillon a été dosé en double.

\section{Résultats - Discussion}

\section{Variation des concentrations basales d'IGFI pour trois chevaux :}

Le suivi des concentrations d'IGF1 pendant 18 heures est résumé dans le tableau 1 et montre que ce paramètre est stable chez les chevaux de sexe différent au cours d'un cycle de 18 heures à la différence de l'hormone de croissance elle-même, aussi bien chez la femelle que l'étalon (6).

\section{Étude de population}

Considérant la population dans son ensemble, la moyenne est de $299 \pm 96 \mathrm{ng} / \mathrm{ml}$ avec un maximum de $550 \mathrm{ng} / \mathrm{ml}$ et un minimum de $55 \mathrm{ng} / \mathrm{ml}$. L'étude des valeurs de concentrations par classe d'âge est donné dans le tableau 2

Tableau I : Étude des concentrations plasmatiques en IGFI au cours du cycle nycthéméral.

\begin{tabular}{|ccccc|}
\hline & Moyenne $(\mathbf{n g} / \mathbf{m l})$ & Écart type $(\mathbf{n g} / \mathbf{m l})$ & Minimum $(\mathbf{n g} / \mathbf{m l})$ & Maximum $(\mathbf{n g} / \mathbf{m l})$ \\
\hline Cheval 1 & 210 & 15 & 165 & 225 \\
Cheval 2 & 279 & 51 & 170 & 279 \\
Cheval 3 & 266 & 36 & 190 & 266 \\
\hline
\end{tabular}

Tableau II : Concentrations plasmatiques en IGF1 par âge ou classe d'âge.

\begin{tabular}{|c|c|c|c|c|c|}
\hline Age ou intervalle & 1 & 2 & $3-4$ & $5-10$ & $11-28$ \\
\hline $\mathrm{n}$ & 27 & 25 & 34 & 35 & 49 \\
\hline Moyenne (ng/ml) & 333 & 349 & 328 & 282 & 248 \\
\hline Écart-type (ng/ml) & 107 & 89 & 87 & 81 & 83 \\
\hline Minimum (ng/ml) & 185 & 245 & 200 & 55 & 160 \\
\hline Maximum (ng/ml) & 449 & 550 & 535 & 465 & 480 \\
\hline
\end{tabular}

Moyenne : $299 \pm 96 \mathrm{ng} / \mathrm{ml}$ 


\section{Concentration d'IGF1 après administration d'hor- mone recombinante équine.}

Après administration d'hormone recombinante équine, chez deux chevaux de 10 et 11 ans, les concentrations maximum observées d'IGF1 étaient respectivement de $708 \mathrm{ng} / \mathrm{ml}$ et $844 \mathrm{ng} / \mathrm{ml}$. Le maximum est observé pendant un temps court. Le retour à des valeurs physiologiques chez ces chevaux apparaît avant la fin de l'administration. Cette étude est en cours d'évaluation chez des chevaux plus jeunes.

L'étude d'autres paramètres est en cours d'investigations : protéines de transport de l'IGF1 principalement les BP1, BP3 et marqueurs osseux comme l'ostéocalcine et la fraction osseuse de la phosphatase alcaline.

Ces résultats sont préliminaires et montrent bien la difficulté de fixer des normes susceptibles de s'appliquer à toute une population, en conséquence il est probable que la solution réside dans un concept multi-factoriel, placé lui même dans le cadre d'un contrôle continu de l'animal. Ce concept est aussi connu sous le vocable de contrôle longitudinal des athlètes.

En conclusion, il paraît évident que le contrôle antidopage est à un carrefour et la route à prendre est longue et coûteuse. Il ne convient pas de renoncer au travail effectué auparavant mais il faut le compléter et l'enrichir avec un concept plus global alliant à la fois toxicologie et biologie endocrinienne.

\section{Remerciements}

Dr P. Delahaut, CER, Laboratoire d'Hormonologie, Marloie, Belgique ; Dr J. Closset, Université de Liège, Belgique ; Dr S. Horning, Thermoquest Bremen, Allemagne ; Pr G. Moneti, CISM, Université de Florence, Italie ; Dr B. Casetta, PE Biosystems, Trieste, Italie ; Dr G. Kearney, Micromass Applicatons Laboratory, Manchester, Grande Bretagne.

\section{Références}

1. De Brabander H.F., De Wasch K., Van Ginkel L.A. et al. Multi-laboratory study of the analysis and kinetics of stanozolol and its metabolites in treated calves. Analyst. $1998 ; 123: 2599-2604$

2. Schänzer W., Delahaut P.H.,. Geyer H., Machnik M., Horning S. Longterm detection of metandienone and stanozolol abuse in athletes by gas chromatography / high resolution mass spectrometryJ. Chromatogr. B. $1996 ; 687$ : 93-108

3. Mück W.M., Henion J.D. High performance liquid chromatography / tandem mass spectrometry: its use for the identification of stanozolol and its major metabolites in human and equine urine. Biomed. Environ. Mass Spectrom. 1990 ; $19: 37-51$

4. Accord International sur l'élevage et les courses et annexes. Fédération Internationale des Autorités Hippiques de Courses au Galop. 46, Place Abel Gance - 92655 Boulogne.

5. Kicman A.T. , Miell J.P., Teale J.D. et al. Serum IGF- 1 and IGF binding proteins 2 and 3 as potential markers of doping with human growth hormone. Clin. Endocr. $1997 ; 47: 43-50$.

6. Thompson Jr D.L., Rahmanian M.S., De Pew C.L. et al. Growth hormone in mares and stallions : Pulsatile secretion response to growth hormonereleasing hormone, and effects of exercise, sexual stimulation and pharmacological agents. J. Anim. Sci. $1992 ; 70: 1201-1207$.

7. Lee P.D.K., Durham S.K., Martinez V. et al. Kinetics of insulin-like growth factor (IGF) and IGF-binding protein responses to a single dose of growth hormone. J. Clin. Endocr. Metab. 1997 ; $82: 2266-2274$.

8. Malinowski K.,. Christensen R.A., Hafs H.D., Scanes C.G. Age and breed differences in thyroid hormones, insulin-like growth factor (IGF)-I and binding proteins in female horses.J. Anim. Sci. $1996 ; 74: 1936-1942$.

9. Thomas M.G., Bennett K., Wimbush P., Keisler D.H., Loch W.C. Plasma concentrations of growth hormone and insulin-like growth factor-I in prepuberal quarter horses and ponies. J. of Equine Vet. Sci. $1998 ; 18: 52-55$. 